\title{
Preliminary investigation of relationship between clinical indicators and CT manifestation patterns of COVID-19 pneumonia improvement
}

\author{
Nannan Shi ${ }^{1 \#}$, Fengxiang Song ${ }^{1 \#}$, Fengjun Liu ${ }^{1}$, Pengrui Song ${ }^{1}$, Yang Lu ${ }^{1}$, Qinguo Hou ${ }^{1}$, Xinyan Hua ${ }^{1}$, \\ Yun Ling ${ }^{2}$, Jiulong Zhang ${ }^{1}$, Chao Huang ${ }^{3,4}$, Lei Shi ${ }^{3,4,5}$, Zhiyong Zhang ${ }^{6}$, Fei Shan ${ }^{1}$, Qi Zhang ${ }^{3,5,7}$, Yuxin Shi ${ }^{1,8}$ \\ ${ }^{1}$ Department of Radiology, ${ }^{2}$ Department of Infectious Disease, Shanghai Public Health Clinical Center, Shanghai, China; ${ }^{3}$ Shanghai Key Laboratory \\ of Artificial Intelligence for Medical Image and Knowledge Graph, Shanghai, China; ${ }^{4}$ Hangzhou YITU Healthcare Technology Co., Ltd., Hangzhou, \\ China; ${ }^{5}$ Shanghai Yizhi Healthcare Technology Co., Ltd., Shanghai, China; ${ }^{6}$ Fudan University, Shanghai, China; ${ }^{7}$ The SMART (Smart Medicine and \\ AI-based Radiology Technology) Lab, School of Communication and Information Engineering, Shanghai University, Shanghai, China; ${ }^{8}$ Shanghai \\ Key Laboratory of Molecular Imaging, Shanghai, China \\ Contributions: (I) Conception and design: N Shi, F Song, Q Zhang, Y Shi; (II) Administrative support: Z Zhang, F Shan; (III) Provision of study \\ materials or patients: Y Ling, F Liu; (IV) Collection and assembly of data: P Song, Y Lu, Q Hou, X Hua; (V) Data analysis and interpretation: J \\ Zhang, C Huang, L Shi; (VI) Manuscript writing: All authors; (VII) Final approval of manuscript: All authors. \\ \#These authors contributed equally to this work. \\ Correspondence to: Qi Zhang, PhD. Shanghai Key Laboratory of Artificial Intelligence for Medical Image and Knowledge Graph, Shanghai 200051, \\ China. Email: qizhang.fdu@gmail.com; Yuxin Shi, MD, PhD. Department of Radiology, Shanghai Public Health Clinical Center, Shanghai 201508, \\ China. Email: shiyuxin@shphc.org.cn.
}

Background: To retrospectively evaluate several clinical indicators related to the improvement of COVID-19 pneumonia on CT.

Methods: A total of 62 patients with COVID-19 pneumonia were included. The CT scores based on lesion patterns and distributions in serial CT were investigated. The improvement and deterioration of pneumonia was assessed based on the changes of CT scores. Grouped by using the temperature, serum lymphocytes and high sensitivity CRP (hs-CRP) on admission respectively, the CT scores on admission, at peak time and at discharge were evaluated. Correlation analysis was carried out between the time to onset of pneumonia resolution on CT images and the recovery time of temperature, negative conversion of viral nucleic acid, serum lymphocytes and hs-CRP.

Results: The CT scores of the fever group and lymphopenia group were significantly higher than those of normal group on admission, at peak time and at discharge; and the CT scores of normal hs-CRP group were significantly lower than those of the elevated hs-CRP group at peak time and at discharge $(\mathrm{P}$ all<0.05). The time to onset of pneumonia resolution on CT image was moderately correlated with negative conversion duration of viral nucleic acid $(\mathrm{r}=0.501, \mathrm{P}<0.05)$ and the recovery time of hs-CPR $(\mathrm{r}=0.496, \mathrm{P}<0.05)$.

Conclusions: COVID-19 pneumonia patients with no fever, normal lymphocytes and hs-CRP had mild lesions on admission, and presented with more absorption and fewer pulmonary lesions on discharge. The negative conversion duration of viral nucleic acid and the recovery time of hs-CPR may be the indicator of the pneumonia resolution.

Keywords: Coronavirus Disease 2019 (COVID-19); computed tomography; pneumonia improvement

Submitted Mar 21, 2020. Accepted for publication Aug 13, 2020.

doi: $10.21037 /$ jtd-20-1420

View this article at: http://dx.doi.org/10.21037/jtd-20-1420 


\section{Introduction}

In December 2019, an increasing number of cases with pneumonia of unknown etiology, now known as Coronavirus Disease 2019 (COVID-19), were found in Wuhan, China. It was caused by severe acute respiratory syndrome coronavirus 2 (SARS-CoV-2), which is a novel $\beta \mathrm{CoV}$ of group $2 \mathrm{~B}$ with $89.1 \%$ nucleotide similarity in genetic sequence to SARS-CoV $(1,2)$. In 2002, the SARS$\mathrm{CoV}$ originated from an animal market in Southern China and then affected more than 8,000 people, with 916 deaths in 29 countries. Subsequently, Middle East respiratory syndrome-CoV (MERS-CoV) affected 2,066 people, with at least 720 deaths in 27 countries $(3,4)$. Compared with the fatal rate of SARS and MERS-CoV, COVID-19 is now the third lethal virus in the coronavirus family. By 10:00 CEST, 22 May 2020, COVID-19 have affected 4,993,470 people, with 327,738 deaths (5).

Computed tomography (CT) provides significant information for early screening, diagnosis and severity assessment of COVID-19. The aim of our study was to explore CT evolution patterns of COVID-19 pneumonia and its relationship with some clinical features, as well as to look for factors related to pneumonia improvement.

\section{Methods}

\section{Patients}

The study was conducted in accordance with the Declaration of Helsinki (as revised in 2013). This study was approved by the Institutional Review Boards of Shanghai Public Health Clinical Center (NO. YJ-2020-S035-01) with a waiver of informed consent.

Our inclusion criteria were: (I) Patients who were confirmed with a positive novel coronavirus nucleic acid antibody (PT-RCR assay with oropharyngeal swabs samples) and admitted to our Center. (II) Discharged patients who had no respiratory symptoms and had two successive (minimum $24 \mathrm{~h}$ sampling interval) negative RTPCR tests for viral RNA with oropharyngeal swabs. (III) Patients received the initial and follow-up thin-section CT examinations; and (IV) CT images demonstrated pneumonia. Thus, a total of 62 patients were included from January 20th 2020 to February 8th 2020.

\section{Image acquisition and evaluation}

SCENARIA 64 CT (Hitachi Medical, Japan) was performed during a single-breath hold at full inspiration, with scanning parameters of $120 \mathrm{kV}$. Low-dose chest smart milliampere (180-400 mA) scanning method was used to reduce the dose. Other settings of the CT imaging protocol were as follows: Advanced Model Iterative reconstruction technique, rotation time: 0.5 second; collimation: $0.625 \mathrm{~mm}$; pitch: 1.5 ; section thickness: $5 \mathrm{~mm}$; matrix: $512 \times 512$. The lung window with a width of $1,200 \mathrm{HU}$ and a level of $-600 \mathrm{HU}$ and the mediastinal window with window width of $350 \mathrm{HU}$ and a window level of $40 \mathrm{HU}$ were set up. Reconstruction was performed by lung smooth with a thickness of $1 \mathrm{~mm}$ and an interval of $0.8 \mathrm{~mm}$.

CT images were evaluated by three experienced thoracic radiologists, who were blinded to the clinical data. The radiologists assessed the lesion patterns including the ground-glass opacity (GGO), which was diagnosed as hazy areas of increased opacity or density without obscuring the underlying airway walls and vessels; consolidation, which was homogeneous opacification of the parenchyma concealing the margins of airway walls and vessels; and the GGO with focal or multifocal areas of consolidation (6).

The CT scores of pulmonary lesions on serial CT were investigated. On the basis of the classification system by Grieser et al. (7), the CT findings were graded on a 3-point scale: Normal attenuation was scored 1, the GGO was scored 2, and the consolidation was scored 3 . The extent of the abnormalities described above was assessed independently for 3 zones in each lung, including the upper (above the level of carina), the middle (between the level of carina and inferior pulmonary vein) and the lower (below the level of inferior pulmonary vein). The extent of each abnormality was determined by visually estimating the percentage (to the nearest $10 \%$ ) of the affected lung parenchyma using the method described. Then the pulmonary parenchyma distribution multiplied by radiological data scores described above. The final data was obtained by adding the scores of the six zones (CT score $=$ CT finding*right upper zone + CT finding*left upper zone + CT finding*right middle zone + CT finding*left middle zone + CT finding*right lower zone + CT finding*left lower zone). The value ranged from 0 to 270 in our study.

The CT scores were acquired and evaluated. The peak time was the time when the CT scores were the highest. When evaluating the CT evolution patterns, the minimum change of ten points in CT scores was considered meaningful. Five patterns of radiographic changes during hospitalization were found: type 1 , progressive radiology improvement; type 2, initial radiographic deterioration to 
Table 1 Clinical characteristics of patients infected with COVID-19 pneumonia

\begin{tabular}{lc}
\hline Clinical characteristics & $\mathrm{N}(\%)$ \\
\hline Gender & $33(53.2)$ \\
Male & $29(46.8)$ \\
Female & $45(16-80)$ \\
Age, median (range)-year & \\
Clinical manifestations & $50(80.6)$ \\
Fever & $20(32.3)$ \\
Cough & $12(19.4)$ \\
Fatigue & $12(19.4)$ \\
Expectoration & $6(9.7)$ \\
Headache & $4(6.5)$ \\
Poor appetite & $4(6.5)$ \\
Sore throat & $3(4.8)$ \\
Stuffy and runny nose & $3(4.8)$ \\
Abdominal distention & $13(6-21)$ \\
Vomit & $1(1.6)$ \\
Basic diseases & $3(4.8)$ \\
Hypertension & $3(4.8)$ \\
Cardiac disease & $1(1.6)$ \\
Hypothyroidism or hyperthyroidism & $1(1.6)$ \\
Chronic liver disease & \\
Depression & \\
\hline & \\
\hline
\end{tabular}

the peak level followed by radiographic improvement; type 3 , fluctuating radiographic changes; type 4, progressive radiographic deterioration; and type 5 , static radiographic appearance.

\section{Statistical analysis}

Statistical analyses were performed using SPSS 25.0 software (IBM, Armonk, NY, USA). For categorical variables, the percentages of patients in each category were calculated. The CT scores between different observers were assessed with intraclass correlation coefficients (ICCs). The CT scores between different groups were compared using the Mann-Whitney U test. Correlations between evolution patterns of chest CT scores and clinical features were analyzed using the $\chi^{2}$ test. The Spearman rank test was used to determine correlations between the time to onset of pneumonia resolution on CT image and the recovery time of temperature, negative conversion of viral nucleic, serum lymphocytes and serum hs-CRP. For all statistical analyses, a p-value less than 0.05 was regarded as statistically significant.

\section{Results}

Sixty-two discharged patients (33 males and 29 females, ages of 16-80 years) were involved in our study. As shown in Table 1 , the most common clinical manifestations were fever $(55 / 62,88.7 \%)$. Other symptoms included cough (20, $32.3 \%)$, fatigue $(12,19.4 \%)$, and expectoration $(12,19.4 \%)$. Twenty patients $(32.3 \%)$ had basic diseases, including hypertension $(12,19.4 \%)$, cardiac disease $(3,4.8 \%)$ and hypothyroidism/hyperthyroidism $(3,4.8 \%)$. The mean hospitalization period was 12 days (range: 6-21 days).

Most of the cases had normal leukocyte count (53/62, $85.5 \%)$, neutrophil count $(51 / 62,82.3 \%)$ and D-dimer (39/62, 62.9\%). Lymphopenia was shown in 29 cases (46.8\%). More than half cases $(35,56.5 \%)$ showed increased serum hs-CRP, while it was normal in 27 cases $(43.5 \%)$. Most cases (53/62, 85.5\%) showed increased erythrocyte sedimentation rate (ESR) (Table 2).

\section{CT imaging findings}

The time from the onset of symptoms to the initial CT examination ranged from 1 to 16 days (mean $6 \pm 3$ days). Good reliability was found between different observers ( $\mathrm{CCC}=0.794)$. On admission, the main abnormalities included GGO (10/62, 16.1\%), GGO with focal or multifocal areas of consolidation (40/62, 64.5\%), and consolidation $(12 / 62,19.4 \%)$. As shown in Table 3, the median CT scores of overall lesions, consolidation and GGO were 100, 60 and 40, respectively. The pulmonary lesions reached to peak in a mean interval of 3 days (range 1-11 days) after initial CT examination on admission. At peak time, the median CT scores of overall lesions, consolidation and GGO were 120, 90 and 20, respectively. At discharge, the median CT scores of overall lesions, consolidation and GGO were 85, 30 and 40, respectively. The CT scores of consolidation were especially reaching significant differences above three key timepoints (Figure 1, on admission vs. at peak, $\mathrm{P}=0.003$; on admission $v s$. at discharge, $\mathrm{P}=0.011$ ).

Among the 62 patients, 9 had twice CT scans, 10 had 
three CT scans, 25 had four CT scans, 14 had five CT scans 3 had six CT scans and the other 1 had seven CT scans. A total of 243 CT scans (mean $4 \pm 1$ ) were acquired and evaluated. A minimum change of ten points in CT scores between two consecutive CT examinations was considered meaningful. We found five patterns of radiographic changes

Table 2 Laboratory parameters of patients infected with COVID-19 pneumonia

\begin{tabular}{lc}
\hline Laboratory parameters & $\mathrm{N}(\%)$ \\
\hline Leukocyte count, $\times 10^{9} / \mathrm{L}$ & $53(85.5)$ \\
Normal $(3.5-9.5)$ & $8(12.9)$ \\
Decreased $(<3.5)$ & $1(1.6)$ \\
Increased $(>9.5)$ & \\
Neutrophil count, $\times 10^{9} / \mathrm{L}$ & $51(82.3)$ \\
Normal $(1.8-6.3)$ & $8(12.9)$ \\
Decreased $(<1.8)$ & $3(4.8)$ \\
Increased $(>6.3)$ & \\
Lymphocyte count, $\times 10^{9} / \mathrm{L}$ & $33(53.2)$ \\
Normal $(1.10-3.2)$ & $29(46.8)$ \\
Decreased $(<1.1)$ & \\
hs-CRP, mg/l & $27(43.5)$ \\
Normal $(0-10)$ & $35(56.5)$ \\
Increased $(>10)$ & \\
D-dimer, $\mu \mathrm{g} / \mathrm{mL}$ & \\
Normal $(0-10)$ & \\
Increased $(>10)$ & \\
Erythrocyte sedimentation rate (ESR), mm/h & \\
Normal $(0-15)$ & $(82.9)$ \\
Increased $(>15)$ & \\
\hline & \\
\hline
\end{tabular}

during hospitalization: type 1 (27/62, 43.5\%), progressive radiology improvement (Figure 2); type 2 (32/62, 51.6\%), initial radiographic deterioration to the peak level followed by radiographic improvement (Figure 3); type $3(1 / 62$, $1.6 \%)$, fluctuating radiographic changes; type 4 (1/62, $1.6 \%)$, progressive radiographic deterioration; and type 5 $(1 / 62,1.6 \%)$, static radiographic appearance (Figure 4$)$.

\section{Relationship between CT evolution and clinical features}

According to the oral temperature on admission, patients were divided into the normal temperature group $\left(<37.3{ }^{\circ} \mathrm{C}\right)$ $(12 / 62,19.4 \%)$ and fever group $\left(\geq 37.3{ }^{\circ} \mathrm{C}\right)(50 / 62,80.6 \%)$. The CT scores of overall lesions in the normal temperature group and fever group were 50 vs. 115 on admission; 85 vs. 140 at peak time; and 50 vs. 100 at discharge $(\mathrm{P}$ all<0.05, Table 4 and Figure 5).

The temperature recovery time of 50 patients was recorded and no correlation was found between the time to onset of pneumonia resolution on CT image and the temperature recovery time $(\mathrm{P}=0.403$, Figure $6 A)$. The negative conversion duration of viral nucleic acid was recorded. The mean time from admission to negative teats for viral nucleic acid was $5 \pm 4$ days (range, $1-18$ days). Pulmonary lesions of 40 (40/62, 64.5\%) patients started to be absorbed after negative conversion of viral nucleic acid within a mean time of 3 days (range, 1-10 days). There was a moderate correlation between negative conversion duration of viral nucleic acid and the time to onset of pneumonia resolution on CT image $(r=0.501, \mathrm{P}<0.05$, Figure $6 B$ ). Only one case in each of type 3,4 and 5 and the time to onset of pneumonia resolution on CT image was not recorded, so they were not included in the correlation study.

According to the lymphocyte counts on admission, they were divided into the normal lymphocyte group $\left(1.10 \times 10^{9}-3.2 \times 10^{9} / \mathrm{L}\right)(33 / 62,53.2 \%)$ and the lymphopenia

Table 3 The CT scores on admission, at peak time and at discharge

\begin{tabular}{|c|c|c|c|c|c|}
\hline & $\begin{array}{l}\text { On admission } \\
\text { The CT scores }\end{array}$ & \multicolumn{2}{|c|}{ At peak } & \multicolumn{2}{|c|}{ At discharge } \\
\hline The CT scores of consolidation & $60(30,120)$ & $90(60,150)$ & $0.003^{*}$ & $30(0,90)$ & $0.011^{*}$ \\
\hline The CT scores of GGO & $40(20,80)$ & $20(0,60)$ & $0.016^{\star}$ & $40(20,60)$ & 0.129 \\
\hline The CT scores of overall lesions & $100(60,140)$ & $120(90,152.5)$ & 0.081 & $85(50,120)$ & $0.029^{\star}$ \\
\hline
\end{tabular}

The CT scores were presented as median (IQR). ${ }^{a}$ Mann-Whitney $U$ test was perform between the CT scores on admission and at peak ( $\left.{ }^{\star} \mathrm{P}<0.05\right) ;{ }^{b}$ Mann-Whitney $\mathrm{U}$ test was perform between the CT scores on admission and at discharge $\left({ }^{*} \mathrm{P}<0.05\right)$. 

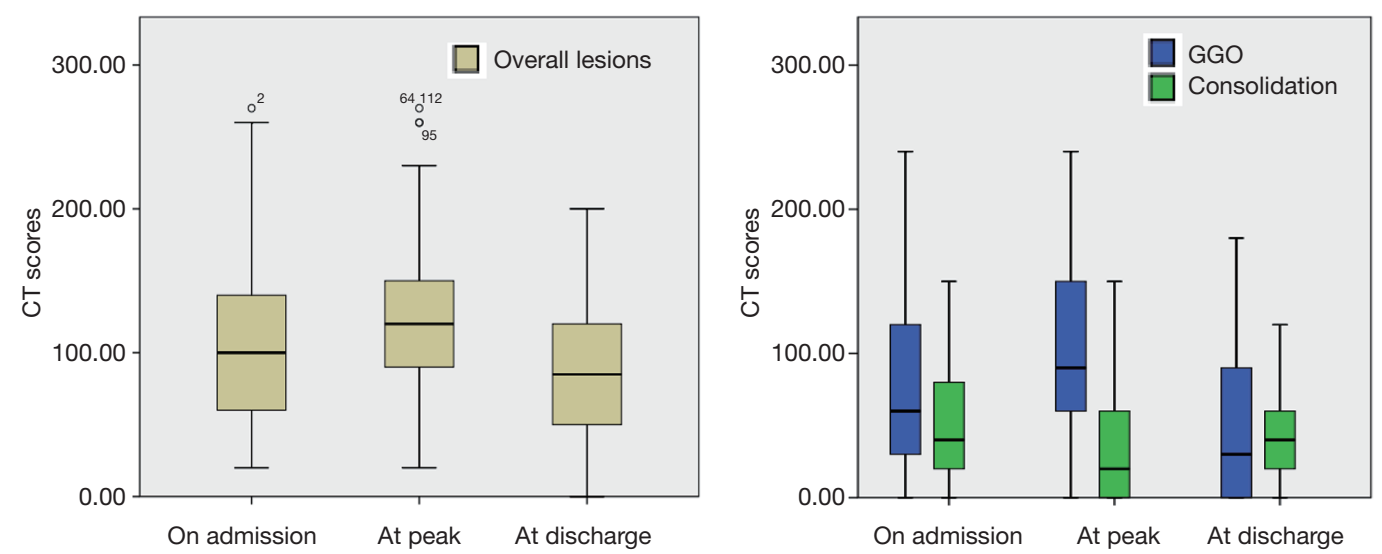

Figure 1 Stacked-bar plot shows the CT scores of GGO and consolidation on admission, at peak time and at discharge.
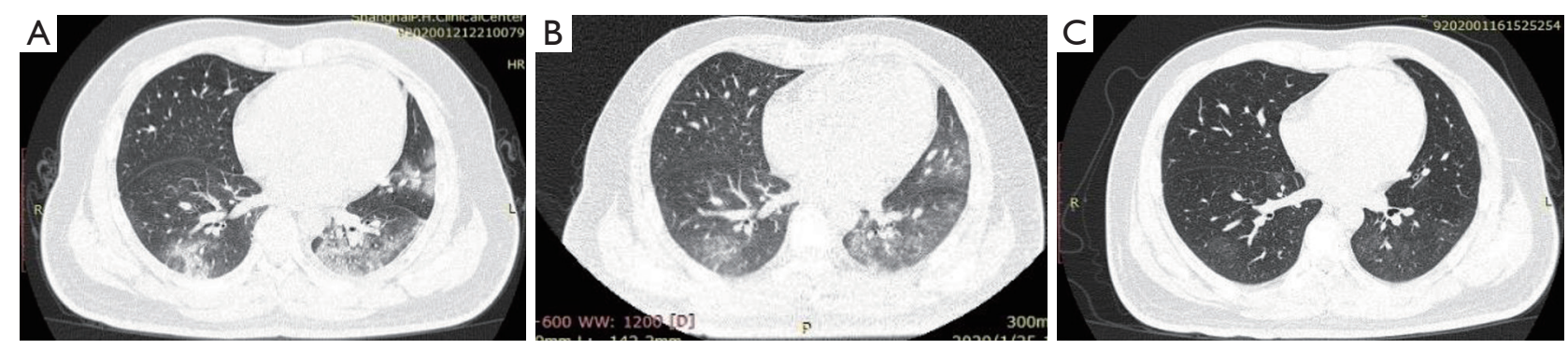

Figure 2 Follow-up CT images of a 32-year-old male with COVID-19 pneumonia, type 1 pattern. (A) On admission (January 21st), GGO with focal or multifocal areas of consolidation was shown in lungs and multiple lobes. (B) After four days of follow-up (January 25 th), partial consolidation became GGO. (C) At discharge (January 29th), GGO was gradually absorbed and the lesions were narrowed.

group $\left(<1.1 \times 10^{9} / \mathrm{L}\right)(29 / 62,46.8 \%)$. On admission, The CT scores of overall lesions in the lymphopenia group were 130 and those in the normal group were $80(\mathrm{P}<0.05)$. At the peak time, the CT scores of overall lesions of the lymphopenia group were 150 and those in the normal group were $100(\mathrm{P}<0.05)$. At discharge, the CT scores of overall lesions of the lymphopenia group were 120 and those of the normal group were $60(\mathrm{P}<0.05)$ (Table 4 and Figure 3). No statistically significant difference was observed in CT evolution patterns between the normal lymphocyte group and the lymphopenia group. Twenty-four patients $(24 / 29,82.8 \%)$ with lymphopenia returned to the normal level before discharge. Analyzing the recovery time from lymphopenia of these 24 patients, it was found that there was no direct correlation between the recovery time from lymphopenia and the time to onset of pneumonia resolution on CT images ( $\mathrm{P}=0.481$, Figure $6 C)$.

According to the hs-CRP counts on admission, the patients were divided into the normal hs-CRP group
$(0-10 \mathrm{mg} / \mathrm{L})$ and the elevated hs-CRP group (>10 mg/L). On admission, the CT score of overall lesions of elevated hs-CRP group were 130 and those of the normal group were 90 . At peak time, the CT scores of overall lesions of the elevated hs-CRP group (140) were significantly higher than those of the normal hs-CRP group $(90)(\mathrm{P}<0.05)$. At discharge, the CT scores of overall lesions of the elevated hs-CRP group (110) were significantly higher than those of the normal hs-CRP group $(60)(\mathrm{P}<0.05)$. The normal hs-CRP group mainly possessed type $1(18 / 25,72 \%)$. The elevated hs-CRP group mainly possessed type $2(25 / 34$, $73.5 \%)(\mathrm{P}<0.05$, Table 5). Thirty-three patients $(33 / 35$, $94.3 \%$ ) with elevated hs-CRP returned to a normal level before discharge. Excluding one type 3 case, the recovery time from evaluated hs-CPR of the left 32 patients was analyzed and it was found that there was a moderate positive correlation between the hs-CPR recovery time and the time to onset of pneumonia resolution on CT images ( $\mathrm{r}=0.496$, $\mathrm{P}<0.05$, Figure 6D). 

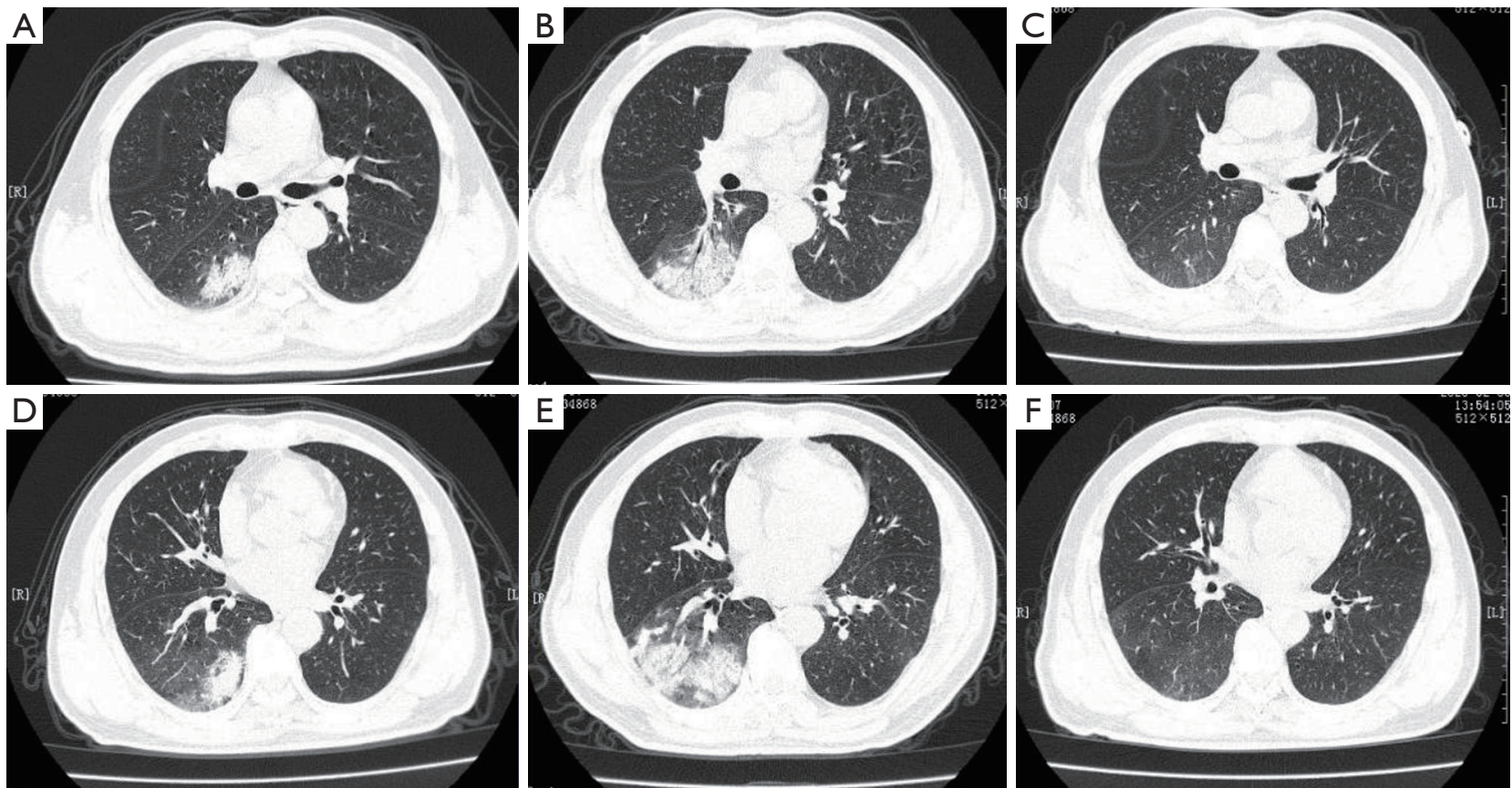

Figure 3 Follow-up CT images of a 63-year-old male with COVID-19 pneumonia, type 2 pattern. (A and D) On admission (January 24th), consolidation was shown in lungs and multiple lobes of a 40-year-old man. (B and E) After five days (January 29th), consolidation was aggravated in progressive stage. (C and F) At discharge (February 6th), GGO was gradually absorbed and the lesions were narrowed.

Type 1
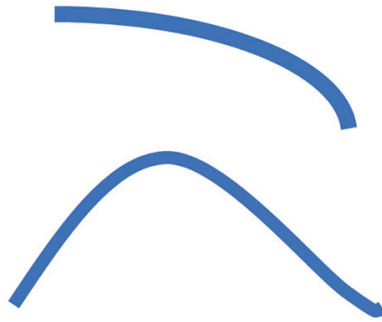

Type 2

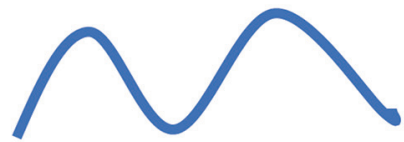

Type 3

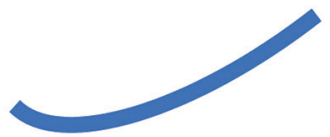

Type 5

Figure 4 Schematic depicts five patterns of CT Evolution Patterns from serial chest CT during hospitalization. 
Table 4 The CT scores and clinical and laboratory parameters

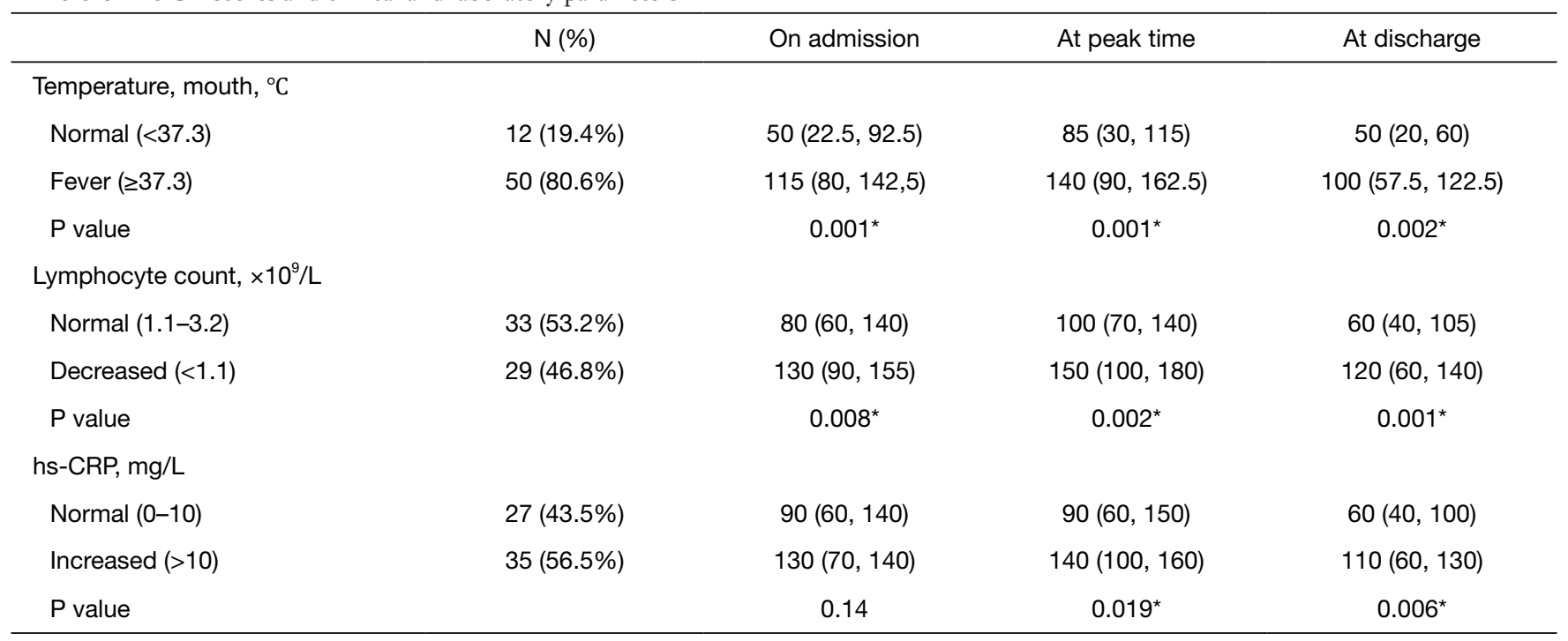

The CT scores were presented as median (IQR). ${ }^{*} \mathrm{P}<0.05$.
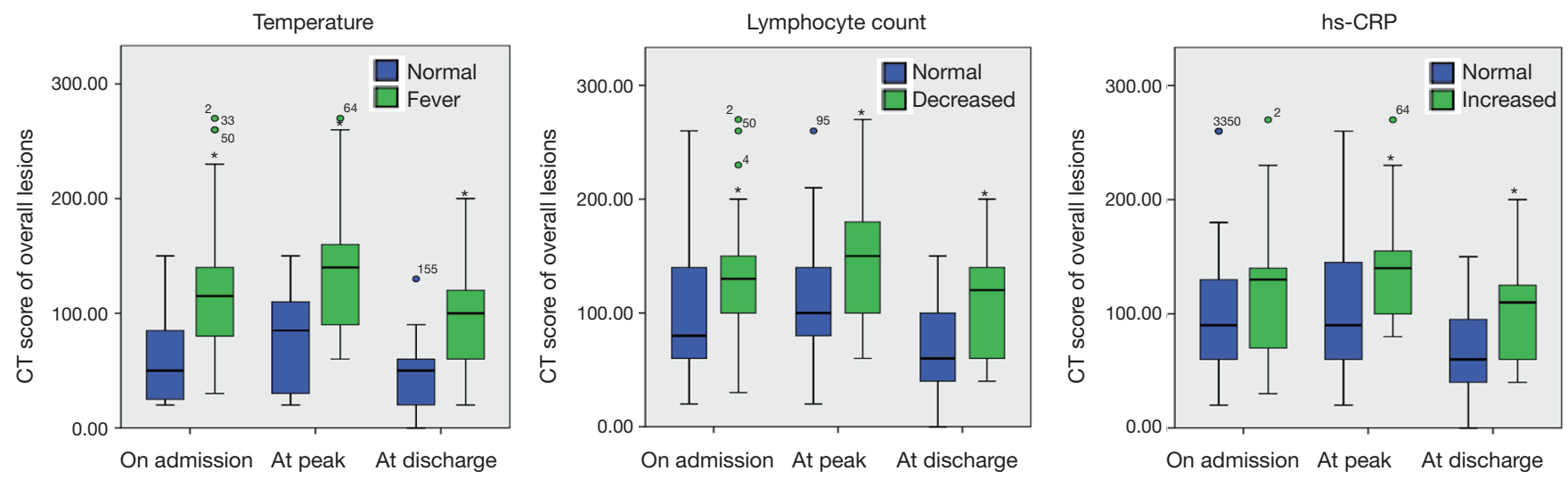

Figure 5 The CT scores of overall lesions and clinical and laboratory parameters $\left({ }^{*} \mathrm{P}<0.05\right)$.

\section{Discussion}

In our study, the CT features of COVID-19 pneumonia were GGO, GGO with focal or multifocal areas of consolidation, and consolidation, which is consistent with recent studies $(8,9)$. The progression of consolidation at peak time and the absorption of consolidation at discharge were more prominent. This result is similar to that of Pan et al., who pointed out that the consolidation was rapidly aggravated in the progressive stage and gradually absorbed in the absorption stage (10). No statistical difference was observed between GGO at different stages. The main reason was that partial GGO converted into consolidation in the progressive stage and partial consolidation converted into GGO in the absorption stage.

Type 1 and type 2 were two main evolution patterns of chest CT scores. However, the duration from onset to admission was longer in type $1(7 \pm 3$ days $)$ than type 2 ( $4 \pm 3$ days), which made the process not completely recorded. This may result in the missing recording of the rising period of the curve. Type 1 could be part of the curve of type 2 , and hence more patients are needed to study the disease course.

According to Diagnosis and Treatment Program of 2019 New Coronavirus Pneumonia (trial sixth version) of the National Health Commission of China, the recovery 

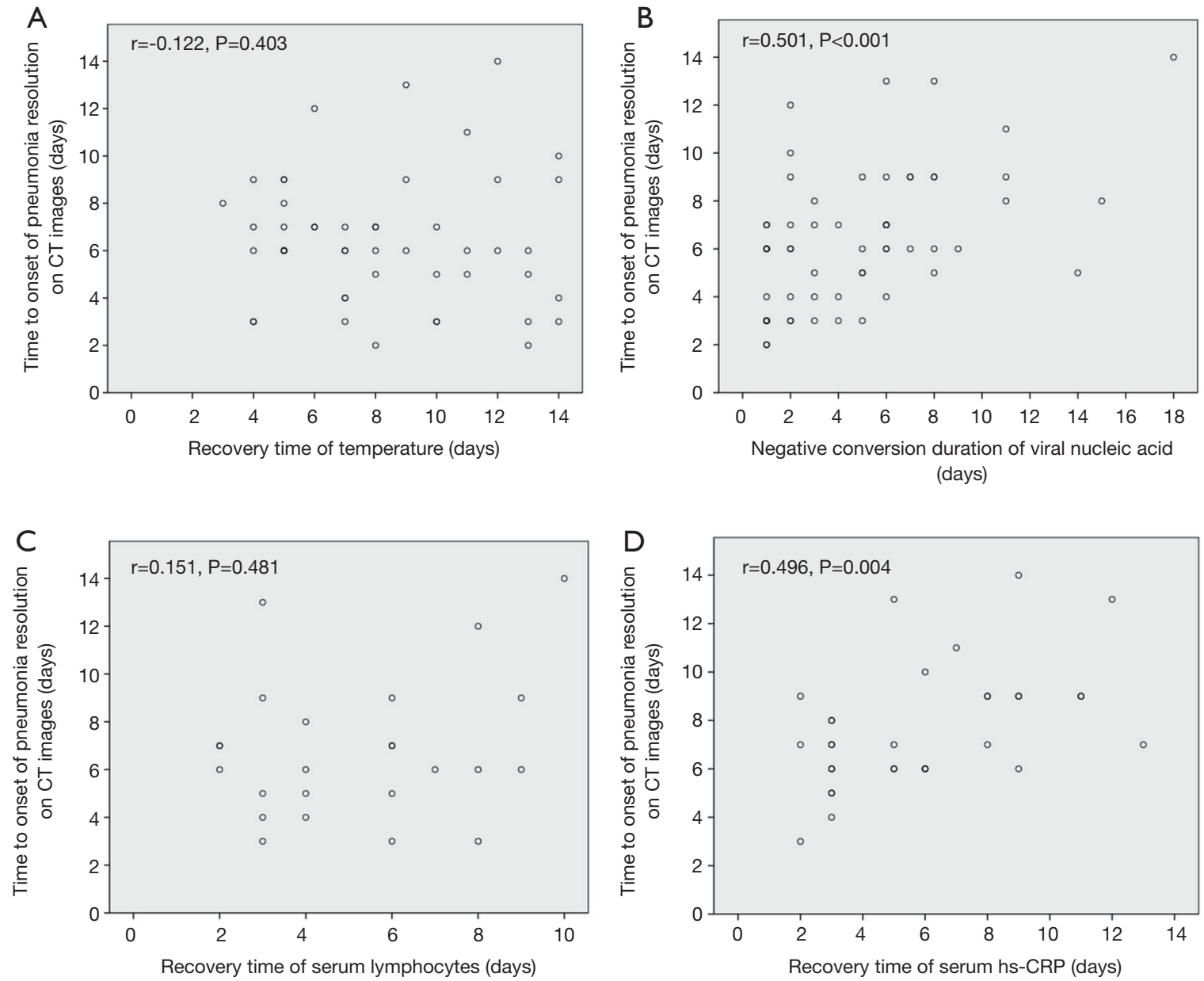

Figure 6 Correlation of the time to onset of pneumonia resolution on CT images with the recovery time of temperature (A), negative conversion of viral nucleic acid (B), serum lymphocytes (C) and hs-CRP (D) using Spearman's correlation coefficient.

Table 5 Patterns of radiographic absorption and clinical and laboratory parameters

\begin{tabular}{lccc}
\hline & Type 1 & Type 2 & P value \\
\hline Temperature & & & \\
Normal $\left(<37.3^{\circ} \mathrm{C}\right)$ & 6 & 4 & \\
Fever $\left(\geq 37.3^{\circ} \mathrm{C}\right)$ & 21 & 28 & 0.448 \\
Lymphocyte count, $\times 10^{\circ} / \mathrm{L}$ & & & \\
Normal $(1.10-3.2)$ & 16 & 15 & \\
Decreased $(<1.1)$ & 11 & 17 & 0.343 \\
hs-CRP, mg/L & & & \\
Normal $(0-10)$ & 18 & 7 & \\
Increased $(>10)$ & 9 & 25 & $0.001^{*}$ \\
\hline
\end{tabular}

${ }^{*} \mathrm{P}<0.05$. of temperature, negative viral nucleic acid and obvious absorption of pneumonia are the elements of discharge criteria. Our results showed the pneumonia absorption on CT was not associated with the temperature recovery. There was a positive correlation between negative conversion duration of viral nucleic acid and the time to onset of pneumonia resolution on CT image. Ling (11) pointed out that the viral RNA of COVID-19 may relate to host cell immunity. So the virus clearance might promote immunity recovery and then accelerate pneumonia absorption. Most cases $(61 / 62,98.4 \%)$ had residual lesions at discharge. Only one mild case had complete absorption of pulmonary lesions at discharge. So, periodic reexamination is important for patients with COVID-19 pneumonia. In SARS, resolution of pneumonitis was delayed in some patients, complete 
resolution occurred after 90 days or more (12).

In our research, the CT scores of normal lymphocyte group were lower than those of the lymphopenia group on admission, at peak time and discharge. The lack of lymphocytes may relate to the severity of pulmonary lesions. This result is consistent with previous studies showing that lymphopenia in COVID-19 infected patients upon hospital admission may predict more severe acute lung injury (13). Chen (14) pointed that monitoring of the variation of lymphocytes for H7N9 patients had important clinical significance, especially for prediction of the resolution of pneumonitis. However, our study did not analyze the effects of different levels of lymphocytes on lung injury because of small sample size and further study of this point is important.

The CT scores of the normal hs-CRP group were less than those of the increased group both at peak time and at discharge. Moreover, the elevated hs-CRP group mainly appeared the initial radiographic deterioration to the peak level followed by radiographic improvement (type 2). In addition, we found that the hs-CPR recovery time was positively related to the onset of pneumonia resolution on CT images. The hs-CRP concentrations in serum will be significantly increased when the body's inflammatory response occurs after infection. In $40 \mathrm{HIV} / \mathrm{HCV}$ coinfected patients, there was a negative correlation between hs-CRP level and CD4+ level. The level of hs-CRP is positively correlated with humoral immune function and negatively correlated with cellular immune function (15). Therefore, the hs-CRP concentrations of COVID-19 infected patients may predict the trend and absorption of pneumonia. However, because of the small sample size, we did not compare the CT scores at different levels of hsCRP, and further research is needed.

There were several limitations in this study. Firstly, the sample size was small. Furthermore, the follow-up CT of patients after discharge has not yet been collected, and therefore the evolution of pulmonary lesions on CT after discharge was not analyzed.

In conclusion, the absorption and development of pneumonia lesions are related to the body temperature and the level of lymphocyte. COVID-19 pneumonia patients with no fever, normal lymphocytes and CRP had mild lesions on admission, and presented with more absorption and fewer pulmonary lesions on discharge. The variation of negative conversion duration of viral nucleic acid and hs-CPR for COVID-19 patients has important clinical relevance, especially for prediction of the resolution of pneumonitis.

\section{Acknowledgments}

Funding: This study was funded by the Science and Technology Commission of Shanghai Municipality (No. 20441900600).

\section{Footnote}

Data Sharing Statement: Available at http://dx.doi. org/10.21037/jtd-20-1420

Peer Review File: Available at http://dx.doi.org/10.21037/jtd20-1420

Conflicts of Interest: All authors have completed the ICMJE uniform disclosure form (available at http://dx.doi. org/10.21037/jtd-20-1420). The authors have no conflicts of interest to declare.

Ethical Statement: The authors are accountable for all aspects of the work in ensuring that questions related to the accuracy or integrity of any part of the work are appropriately investigated and resolved. The study was conducted in accordance with the Declaration of Helsinki (as revised in 2013). The study was approved by This study was approved by the Institutional Review Boards of Shanghai Public Health Clinical Center (NO. YJ-2020-S035-01) with a waiver of informed consent.

Open Access Statement: This is an Open Access article distributed in accordance with the Creative Commons Attribution-NonCommercial-NoDerivs 4.0 International License (CC BY-NC-ND 4.0), which permits the noncommercial replication and distribution of the article with the strict proviso that no changes or edits are made and the original work is properly cited (including links to both the formal publication through the relevant DOI and the license). See: https://creativecommons.org/licenses/by-nc-nd/4.0/.

\section{References}

1. Gao QY, Chen YX, Fang JY. 2019 novel coronavirus infection and gastrointestinal tract. J Dig Dis 2020;21:125-6.

2. Liu Z, Xiao X, Wei X, et al. Composition and divergence of coronavirus spike proteins and host ACE2 receptors 
predict potential intermediate hosts of SARS-CoV-2. J

Med Virol 2020;92:595-601.

3. Yin Y, Wunderink RG. MERS, SARS and other coronaviruses as causes of pneumonia. Respirology 2018;23:130-7.

4. Phan T. Novel coronavirus: From discovery to clinical diagnostics. Infect Genet Evol 2020;79:104211.

5. WHO. Coronavirus disease (COVID-19) Situation Report - 123. Available online: https://www.who.int/docs/defaultsource/coronaviruse/situation-reports/20200522-covid19-sitrep-123.pdf?sfvrsn=5ad1bc3

6. Feng F, Jiang Y, Yuan M, et al. Association of radiologic findings with mortality in patients with avian influenza H7N9 pneumonia. PLoS One 2014;9:e93885.

7. Grieser C, Goldmann A, Steffen IG, et al. Computed tomography findings from patients with ARDS due to Influenza A (H1N1) virus-associated pneumonia. Eur J Radiol 2012;81:389-94.

8. Kanne JP. Chest CT Findings in 2019 Novel Coronavirus (2019-nCoV) Infections from Wuhan, China: Key Points for the Radiologist. Radiology 2020;295:16-7.

9. Song F, Shi N, Shan F, et al. Emerging Coronavirus 2019-

Cite this article as: Shi N, Song F, Liu F, Song P, Lu Y, Hou Q, Hua X, Ling Y, Zhang J, Huang C, Shi L, Zhang Z, Shan F, Zhang Q, Shi Y. Preliminary investigation of relationship between clinical indicators and CT manifestation patterns of COVID-19 pneumonia improvement. J Thorac Dis 2020;12(10):5896-5905. doi: 10.21037/jtd-20-1420
nCoV Pneumonia. Radiology 2020;295:210-7.

10. Pan F, Ye T, Sun P, et al. Time Course of Lung Changes On Chest CT During Recovery From 2019 Novel Coronavirus (COVID-19) Pneumonia. Radiology 2020;295:715-21.

11. Ling $Y, X u S$, Lin $Y$, et al. Persistence and clearance of viral RNA in 2019 novel coronavirus disease rehabilitation patients. Chin Med J (Engl) 2020;133:1039-43.

12. Wang CH, Liu CY, Wan YL, et al. Persistence of lung inflammation and lung cytokines with high-resolution CT abnormalities during recovery from SARS. Respir Res 2005;6:42.

13. Liu Y, Yang Y, Zhang C, et al. Clinical and biochemical indexes from 2019-nCoV infected patients linked to viral loads and lung injury. Sci China Life Sci 2020;63:364-74.

14. Chen C, Chen J, Huang JA. Persistence of lymphocytopenia with CT abnormalities among patients with critical H7N9 swine-origin influenza A virus infection. Jpn J Radiol 2015;33:657-62.

15. Dong Y, Zhi X, Lei G. Changes of body immunity and inflammatory response in HIV/HCV co-infected patients. Exp Ther Med 2019;17:403-7. 